\title{
Networked Control System Design for Turbofan Aeroengines with Aging and Deterioration
}

\author{
Ruichao Li $\mathbb{D}$, ${ }^{1}$ Sing Kiong Nguang $\mathbb{D}^{2},{ }^{2}$ Yingqing Guo $\mathbb{D}^{1},{ }^{1}$ and Yifeng Chen $\mathbb{D}^{1}$ \\ ${ }^{1}$ Northwestern Polytechnical University, Xi'an, Shaanxi, China \\ ${ }^{2}$ The University of Auckland, Auckland, New Zealand
}

Correspondence should be addressed to Ruichao Li; liruichao@mail.nwpu.edu.cn

Received 30 May 2018; Accepted 19 August 2018; Published 11 October 2018

Academic Editor: Yan-Ling Wei

Copyright (C) 2018 Ruichao Li et al. This is an open access article distributed under the Creative Commons Attribution License, which permits unrestricted use, distribution, and reproduction in any medium, provided the original work is properly cited.

\begin{abstract}
This paper is concerned with designing a networked controller for a mixed flow two-spool turbofan aeroengine with aging and deterioration. Firstly, the state-space representation of the aeroengine considering aging and deterioration is identified, by which the engine system with aging and deterioration is modeled as an uncertain linear system. Then based on this uncertain linear system, theoretical results from the networked control systems and the regional pole assignment are introduced to formulate the networked engine control design in the form of linear matrix inequalities (LMIs). By solving these LMIs simultaneously, a networked engine controller is obtained which guarantees both the robustness against delay/dropout and the satisfactory dynamic performance. Finally, the proposed method is applied to an aerothermodynamic component-level engine simulator to demonstrate its validity and applicability. The corresponding delay/dropout margin is also calculated, which provides reference for the future development of the distributed engine control system.
\end{abstract}

\section{Introduction}

The next revolution in turbine engine control systems would be the physical distribution of control functionality, known as distributed engine control (DEC) [1]. The concept of DEC is borrowed from the distributed control system in automated industry, which is featured by the application of advanced data buses, smart sensors, and smart actuators $[2,3]$. The realization of DEC is beneficial to the implementation of the advanced control mode and control algorithm, such as active control, fuzzy control, and model-based control, which bring advantages of weight reduction, modularity, high reliability, high intelligence, etc. [4-10].

However, due to immatureness of the high-temperature electronics $[11,12]$, transition from the centralized control system to the fully distributed control system will be a gradual process. An intermediate distributed system approach is called the networked engine control (NEC) system, in which the data bus is introduced to replace the analog signal transmission. The A/D conversion functions (sampling, shaping, and quantization) of the centralized controller are moved into smart sensors while the control logic still remains as the central form (single controller).

Due to the introduction of the data bus, transmission delay and packet dropout are inevitable in a NEC system. Depending on how network-induced delays and packet dropouts are handled, a number of methods are developed on stability analysis and control design for networked control systems (NCSs). Among them, time-delay system approach and switched system approach are the two mainstream methods. The time-delay system approach yue 2004 state, Jiang 2008 A, e.g., $[13,14]$, models the packet dropout as input-delay, and therefore, the maximum allowable number of consecutive packet dropouts is calculated. The switched system approach models the NCS as a switched system with arbitrary switching Zhang 2008 modelling, Zhang 2009 robust, Donkers 2011 stability, Kruszewski 2012 switched, e.g., [15-18], or known probability switching $[19,20]$, which is usually applied in the discrete-time domain.

As a safety-critical power device, it is crucial to figure out to what degree the control system could tolerate the delay and the packet dropout. To settle this problem, different 
kinds of NEC system models are established by scholars in the aeroengine control field. Constant delay and deterministic dropout are considered by $[21,22]$ who calculate the worst case bound on the number of consecutive dropouts. The case of stochastic dropout has also been considered. Reference [23] models the packet dropout as a Bernoulli process and calculates the maximum allowable packet dropping probability for the decentralized NEC system. Random delays in the NEC system are considered by [24, 25].

Although a lot of work has been done on the NEC systems, the requirement of the dynamic performance has not been fully considered. Since previous results are developed based on the Lyapunov stability theory, the overall system is Lyapunov stable under the networkinduced factors but it may still have a very small decay rate if no constraints are imposed. Besides, the effect of the uncertainty has also not been considered in the design stage; thus, previous results are still far away from practical application. Based on the discussion above, the goal of this paper is to design a NEC system satisfying the following control requirements:

(1) Robustness against the transmission delay and packet dropout

(2) Satisfactory dynamical performance under different degrees of aging and deterioration

(3) Zero tracking error

To satisfy these control requirements, theoretical results from the networked control systems and the regional pole placement $[26,27]$ which have been studied previously are considered as solutions. In this paper, these theories are further extended to an uncertain case and formulated in the form of LMIs which are numerically tractable. Besides, efforts are also made to establish the uncertain description for aeroengines to make the proposed method applicable.

This paper is organized as follows. Section 2 introduces the modeling process of the aeroengine. Section 3 presents the stability theory and controller design of the NEC system. In Section 4, the robust networked controller and its corresponding delay/dropout margin are calculated. Then simulations are performed to demonstrate the effectiveness of the proposed method. Discussions are also extended on further simulation results. Finally, Section 5 presents conclusions. The symbols used in this paper are listed in Table 1.

\section{Modeling of the Aeroengine}

In this section, the working principle of the two-spool turbofan aeroengine and its commercial simulator is introduced first. Then by treating the complex simulator as a black box, a group of linear models is identified. These linear models are further redescribed as a nominal model with norm-bounded uncertainty to facilitate the controller design.
TABLE 1: Nomenclature.

\begin{tabular}{|c|c|c|}
\hline Symbol & Type & Meaning \\
\hline\|\| & Function & Euclidean vector norm \\
\hline * & Function & Ellipsis for the symmetric terms \\
\hline $\operatorname{diag}(\cdots)$ & Function & Yielding a block diagonal matrix \\
\hline $\operatorname{sym}(\mathbf{A})$ & Function & Denoting $\mathbf{A}+\mathbf{A}^{\mathrm{T}}$ \\
\hline I & Matrix & $\begin{array}{l}\text { Identity matrix of } \\
\text { appropriate dimensions }\end{array}$ \\
\hline$u, x, y, w$ & Vector & $\begin{array}{l}\text { Input, state, output, } \\
\text { perturbation }\end{array}$ \\
\hline$x_{s s}, y_{s s}, u_{s s}, w_{s s}$ & Vector & $\begin{array}{l}\text { Steady-state values } \\
\quad \text { of } u, x, y, w\end{array}$ \\
\hline$\delta u, \delta x, \delta y$ & Vector & $\begin{array}{c}\text { Deviation between } \\
u, x, y \text { and } x_{s s}, y_{s s}, u_{s s}\end{array}$ \\
\hline$u_{i}, x_{i}, y_{i}, w_{i}$ & Scalar & The ith element in $u, x, y, w$ \\
\hline$z$ & Vector & State of the integrator \\
\hline $\bar{x}$ & Vector & Augmented state \\
\hline $\mathscr{A}, \mathscr{B}, \mathscr{C}, \mathscr{D}$ & Matrix & Identified system matrix \\
\hline$\overline{\mathscr{A}}, \overline{\mathscr{B}}$ & Matrix & $\begin{array}{l}\text { Augmented identified } \\
\text { system matrix }\end{array}$ \\
\hline $\mathscr{A}_{l}, \mathscr{B}_{l}, \mathscr{C}_{l}, \mathscr{D}_{l}$ & Matrix & $\begin{array}{l}\text { Identified system matrix } \\
\text { under the } l \text { th health condition }\end{array}$ \\
\hline$\overline{\mathscr{A}}_{l}, \overline{\mathscr{B}}_{l}$ & Matrix & $\begin{array}{l}\text { Augmented identified } \\
\text { system matrix }\end{array}$ \\
\hline A, B, C, D & Matrix & Nominal system matrix \\
\hline$\Delta \mathbf{A}, \Delta \mathbf{B}, \Delta \mathbf{C}, \Delta \mathbf{D}$ & Matrix & Norm-bounded uncertainty \\
\hline $\mathbf{K}_{x}, \mathbf{K}_{z}, \mathbf{K}$ & Matrix & Feedback gain matrix \\
\hline $\mathscr{L}, \mathscr{M}$ & Set & $\begin{array}{l}\text { Set whose members contain } \\
\text { a group of } \mathscr{A}_{l}, \mathscr{B}_{l}, \mathscr{C}_{l}, \mathscr{D}_{l}\end{array}$ \\
\hline$\overline{\mathscr{L}}, \overline{\mathscr{M}}$ & Set & $\begin{array}{c}\text { Set whose members contain } \\
\text { a group of } \overline{\mathscr{A}}_{l}, \overline{\mathscr{B}}_{l}\end{array}$ \\
\hline $\mathcal{N}$ & Matrix & Interval matrix \\
\hline $\mathbf{G}_{i}, \mathbf{E}_{i}(i \in\{a, b, c, d\})$ & Matrix & Constant matrix \\
\hline$\overline{\mathbf{G}}, \overline{\mathbf{E}}_{a}, \overline{\mathbf{E}}_{b}$ & Matrix & Augmented constant matrix \\
\hline $\mathbf{F}_{i}(i \in\{a, b, c, d\})$ & Matrix & $\begin{array}{l}\text { Uncertain matrix } \\
\text { satisfying } \mathbf{F}_{\mathrm{i}}^{\mathrm{T}} \mathbf{F}_{i} \leq \mathbf{I}\end{array}$ \\
\hline$\overline{\mathbf{F}}$ & Matrix & $\begin{array}{l}\text { Augmented uncertain } \\
\text { matrix satisfying } \overline{\mathbf{F}}^{\mathrm{T}} \overline{\mathbf{F}} \leq \mathbf{I}\end{array}$ \\
\hline$h$ & Scalar & Sampling period \\
\hline$\eta$ & Scalar & Delay/dropout margin \\
\hline$\tau_{\max }$ & Scalar & $\begin{array}{l}\text { Maximum allowable } \\
\text { transmission delay }\end{array}$ \\
\hline$n_{\max }$ & Scalar & $\begin{array}{l}\text { Maximum allowable number } \\
\text { of consecutive packet dropouts }\end{array}$ \\
\hline $\mathscr{C}(-q, r)$ & Set & $\begin{array}{l}\text { A disk region of which the } \\
\text { center is }-q \text { and the radii is } r\end{array}$ \\
\hline
\end{tabular}

2.1. Mixed Flow Two-Spool Turbofan Aeroengine. The crosssection of a mixed flow two-spool turbofan aeroengine is given in Figure 1. In a two-spool aeroengine, the compressors 


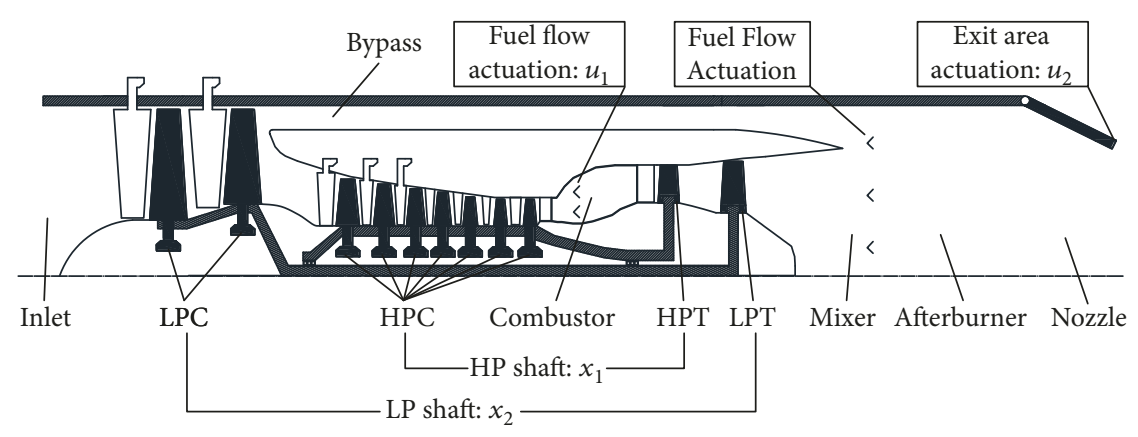

Figure 1: Cross-section of a mixed flow two-spool turbofan aeroengine.

TABLE 2: Structure and physical meaning of the identified model.

\begin{tabular}{lccc}
\hline Signal type & Notation & Designed value & Physical meaning \\
\hline Input $u$ & $u_{1}$ & $5813 \mathrm{~kg} / \mathrm{h}$ & Main combustor fuel flow \\
& $u_{2}$ & $0.26 \mathrm{~m}^{2}$ & Nozzle exit area \\
State $x$ & $x_{1}$ & $8880 \mathrm{rpm}$ & Rotation speed of the LP shaft \\
& $x_{2}$ & $16419 \mathrm{rpm}$ & Rotation speed of the HP shaft \\
Output $y$ & $y_{1}$ & $8880 \mathrm{rpm}$ & Being the same with $x_{1}$ \\
Health parameter $w$ & $w_{1}, \ldots, w_{8}$ & $1.00, \ldots, 1.00$ & Flow modifiers and efficiency modifiers of the LPC, HPC, HPT, and LPT \\
\hline
\end{tabular}

and turbines are linked by concentric shafts which rotate independently, namely, the low-pressure compressor (LPC) is driven by the low-pressure turbine (LPT) and the highpressure compressor (HPC) is powered by the highpressure turbine (HPT).

In [28], using GasTurb modeling software, an aerothermodynamic component-level aeroengine simulator is established for the mixed flow two-spool turbofan aeroengine with realistic structural parameter and features of compressors/turbines. The turbine engine modeling software, GasTurb, is a commercial software $[29,30]$ which is widely used for modeling and simulating gas turbine aeroengines. For the convenience in controller design, the aeroengine simulator developed in [28] is complied under the MATLAB/ Simulink environment further. However, due to the limited space and the modeling of simulators being not the main focus of the paper, the modeling process of the simulator is not included in this paper. Readers who are interested could obtain a general knowledge about the modeling process from the gas turbine textbook (see chap. 2.5 of $[28,31]$ ).

The nonlinear state and output equations of the aeroengine simulator are expressed as

$$
\left\{\begin{array}{l}
\dot{x}=f(x, u, w) \\
y=g(x, u, w)
\end{array}\right.
$$

where $f$ and $g$ are nonlinear functions of the state vector $x$, the input vector $u$, and the health parameter vector $w$ as given in Table 2. The degradation is due to usage and aging results in the higher fuel consumption and exhaust gas temperature, which further yields a shorter component life and a higher cost. Generally, the degradation is reflected as changes in flow characteristics and efficiencies of the rotational components. Therefore, a group of multipliers, which are referred as "health parameters," are introduced. For instance, $w_{i}=1(i=1, \ldots, 8)$ denotes a new component while a value being lower than 1 denotes a degraded one. For the engine which needs an overhaul, the health parameters may degrade to a maximum of 0.99 .

2.2. State-Space Models Obtained by the System Identification. Considering the complexity of the simulator, the system identification technique is utilized to obtain a state-space representation. At the steady-state operating point $\left(x_{s s}, y_{s s}\right.$, $\left.u_{s s}, w_{s s}\right)$, the simulator is identified as

$$
\left\{\begin{array}{l}
\dot{x}(t)=\mathscr{A} \cdot \delta x(t)+\mathscr{B} \cdot \delta u(t), \\
\delta y(t)=\mathscr{C} \cdot \delta x(t)+\mathscr{D} \cdot \delta u(t),
\end{array}\right.
$$

where symbol $\delta$ denotes the deviation from the steadystate point. 

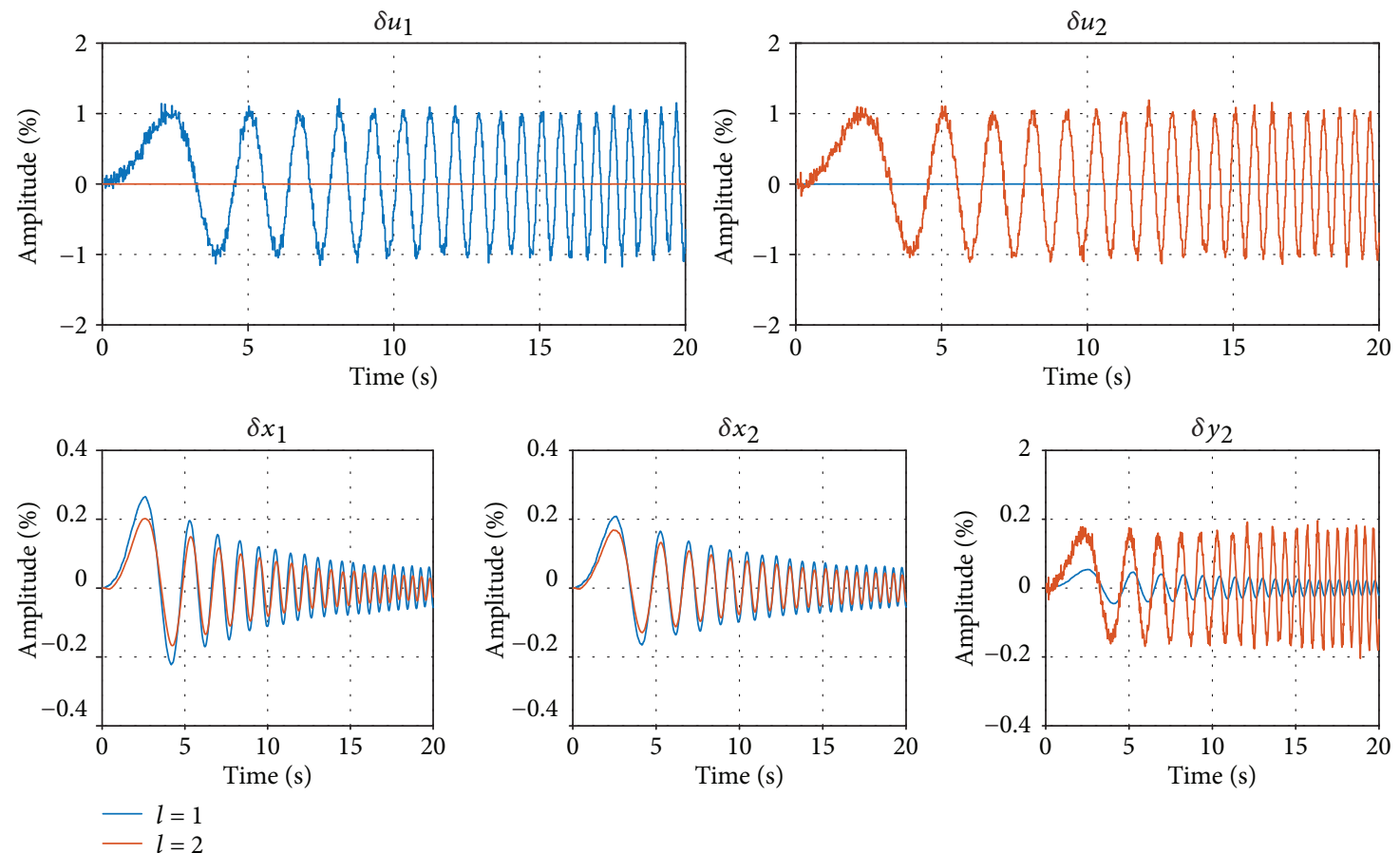

FIgUre 2: Data generated for the system identification.

Take the "full health" status $w=[1, \ldots, 1]^{\mathrm{T}}$ as an example. To apply the system identification technology, outputs of the aeroengine simulators $y_{1}$ and $y_{2}$ are first driven to their designed value given in Table 2; then two excitation signals $\delta u^{l}(t)(l=1,2)$ are added separately to the input channels to generate $\delta x^{l}(t)$ and $\delta y^{l}(t)(l=1,2)$. These two excitation signals are taken, respectively, as

$$
\begin{aligned}
& \delta u^{1}(t)=\left[\begin{array}{c}
\operatorname{chirp}(t) \\
0
\end{array}\right]^{\mathrm{T}}+\omega(t), \\
& \delta u^{2}(t)=\left[\begin{array}{c}
0 \\
\operatorname{chirp}(t)
\end{array}\right]^{\mathrm{T}}+\omega(t),
\end{aligned}
$$

where $\operatorname{chirp}(t)$ is a swept-frequency cosine signal with amplitude being $1 \% u_{\text {designed }}$. As stated in chap. 3.1 of [31], frequencies of the dominant dynamics are generally not higher than $2 \mathrm{~Hz}$ for aeroengines. Therefore, the frequency of $\operatorname{chirp}(t)$ is set to increase linearly from 0 to $2 \mathrm{~Hz}$ within $20 \mathrm{~s}$, which is adequate to extract the spectral characteristics of rotors. Moreover, zero-mean white Gaussian noises $\omega(t)$ are also added to the excitation signals such that the signal-to-noise ratio (SNR) satisfies

$$
\mathrm{SNR}=\frac{\sigma_{\text {signal }}^{2}}{\sigma_{\text {noise }}^{2}}=100
$$

in which $\sigma$ denotes the standard deviation.
The excitation signals $\delta u^{l}=\left[u_{1}^{l}(t), u_{2}^{l}(t)\right]^{\mathrm{T}}(l=1,2)$ and the deviations $\delta x_{1}^{l}, \delta x_{2}^{l}$, and $\delta y_{2}^{l}(l=1,2)$ generated from the aeroengine simulator are depicted in Figure 2, which have already been normalized by their designed value. With these two samples, the system matrices $(\mathscr{A}, \mathscr{B}, \mathscr{C}, \mathscr{D})$ under the "full-health" status are identified by the method proposed in [32] as

$$
\begin{aligned}
& \mathscr{A}=\left[\begin{array}{cc}
-5.792 & 4.818 \\
0.612 & -4.390
\end{array}\right], \\
& \mathscr{B}=\left[\begin{array}{cc}
0.628 & 0.363 \\
0.734 & 0.579
\end{array}\right], \\
& \mathscr{C}=\left[\begin{array}{cc}
1 & 0 \\
-0.578 & 1.900
\end{array}\right], \\
& \mathscr{D}=\left[\begin{array}{cc}
0 & 0 \\
0.033 & 0.707
\end{array}\right],
\end{aligned}
$$

For simplicity, it is assumed that all health parameters $w_{i}(i=1, \ldots, 8)$ degrade simultaneously. Then by varying $w_{i}$ from 1 to 0.99 evenly and still maintaining $y$ at its designed value $y_{\text {designed }}$ given in Table 3 , a group of matrices $\mathscr{A}_{l}, \mathscr{B}_{l}, \mathscr{C}_{l}$, and $\mathscr{D}_{l}(l=1,2, \ldots, 11)$ is obtained via the system identification method above, which constitutes the set $\mathscr{L}$ below

$$
\mathscr{L} \triangleq\left\{\Lambda \mid \Lambda=\left[\begin{array}{cc}
\mathscr{A}_{l} & \mathscr{B}_{l} \\
\mathscr{C}_{l} & \mathscr{D}_{l}
\end{array}\right], \quad l=1,2, \ldots, 11\right\} .
$$


TABLE 3: Delay/dropout margin of each disk region $(r=0.6 q)$.

\begin{tabular}{lcccccccc}
\hline$-q$ & -6 & -7 & -8 & -9 & -10 & -11 & -12 & -13 \\
$\eta_{\max }$ & 0.143 & 0.121 & 0.104 & 0.090 & 0.079 & 0.070 & 0.062 & 0.056 \\
$-q$ & -14 & -15 & -16 & -17 & -18 & -19 & -20 & \\
$\eta_{\max }$ & 0.050 & 0.046 & 0.041 & 0.038 & 0.034 & 0.031 & 0.028 & \\
\hline
\end{tabular}

Due to the limited space, the values of these system matrices are not listed here.

2.3. Derivation of the Norm-Bounded Uncertainty. In this paper, degradations of the health parameters $w$ (representing aging and deterioration) are viewed as the source of uncertainties for the identified state-space models. The degradation has two adverse effects on the identified linear models: On the one hand, the degradation is reflected in the drift of the steady-state value, and on the other hand, it causes the perturbation of the system matrices.

Since the identified system matrices $\mathscr{A}, \mathscr{B}, \mathscr{C}$, and $\mathscr{D}$ vary with $w$, they are supposed to be decomposed as

$$
\begin{aligned}
& \mathscr{A}=\mathbf{A}+\Delta \mathbf{A}, \\
& \mathscr{B}=\mathbf{B}+\Delta \mathbf{B},
\end{aligned}
$$

$$
\mathscr{M} \triangleq\left\{\Lambda \mid \Lambda=\left[\begin{array}{cc}
\mathbf{A} & \mathbf{B} \\
\mathbf{C} & \mathbf{D}
\end{array}\right]+\left[\begin{array}{cc}
\mathbf{G}_{a} \mathbf{F}_{a} \mathbf{E}_{a} & \mathbf{G}_{b} \mathbf{F}_{b} \mathbf{E}_{b} \\
\mathbf{G}_{c} \mathbf{F}_{c} \mathbf{E}_{c} & \mathbf{G}_{d} \mathbf{F}_{d} \mathbf{E}_{d}
\end{array}\right], \quad \mathbf{F}_{i}^{\mathrm{T}} \mathbf{F}_{i} \leq \mathbf{I}, i \in\{a, b, c, d\}\right\} .
$$

$$
\begin{aligned}
& \mathscr{C}=\mathbf{C}+\Delta \mathbf{C}, \\
& \mathscr{D}=\mathbf{D}+\Delta \mathbf{D},
\end{aligned}
$$

in which $\Delta \mathbf{A}, \Delta \mathbf{B}, \Delta \mathbf{C}$ and $\Delta \mathbf{D}$ are norm-bounded uncertainties satisfying

$$
\begin{aligned}
& \Delta \mathbf{A}=\mathbf{G}_{a} \mathbf{F}_{a} \mathbf{E}_{a}, \\
& \Delta \mathbf{B}=\mathbf{G}_{b} \mathbf{F}_{b} \mathbf{E}_{b}, \\
& \Delta \mathbf{C}=\mathbf{G}_{c} \mathbf{F}_{c} \mathbf{E}_{c}, \\
& \Delta \mathbf{D}=\mathbf{G}_{d} \mathbf{F}_{d} \mathbf{E}_{d},
\end{aligned}
$$

where $\mathbf{G}_{i}$ and $\mathbf{E}_{i}(i \in\{a, b, c, d\})$ are constant matrices of appropriate dimensions. $\mathbf{F}_{i}$ is the uncertain matrix with Lebesgue measurable elements that satisfy $\mathbf{F}_{i}^{\mathrm{T}} \mathbf{F}_{i} \leq \mathbf{I}$.

When obtaining the uncertain state-space representation (7), the difficulty lies in how to decompose a group of matrices $\mathscr{A}_{l}$ further into a uniform norm-bounded description $\mathbf{A}+\mathbf{G}_{a} \mathbf{F}_{a} \mathbf{E}_{a}$, namely, transforming $\mathscr{L}$ to the set $\mathscr{M}$ defined below.
To the best of our knowledge, there exists no transformation method such that $\mathscr{L}$ equals $\mathscr{M}$.

Therefore, efforts are made to find a set $\mathscr{M}$ which could encompass $\mathscr{L}$, namely, $\mathscr{L} \subseteq \mathscr{M}$. This is handled via the concept of interval matrix introduced below.

Definition 1 [33]. With the given matrices $\mathbf{P} \triangleq\left(p_{i j}\right)_{n \times n}, \mathbf{Q} \triangleq$ $\left(q_{i j}\right)_{n \times n}$, and $\mathscr{A} \triangleq\left(a_{i j}\right)_{n \times n}$, notation $\mathcal{N}[\mathbf{P}, \mathbf{Q}]$ is used to denote the set of matrix $\mathscr{A}$ which satisfies $p_{i j} \leq a_{i j} \leq q_{i j}$, namely,

$$
\mathcal{N}[\mathrm{P}, \mathrm{Q}] \triangleq\left\{\mathscr{A} \in \mathbb{R}^{n \times n} \mid p_{i j} \leq a_{i j} \leq q_{i j}, \quad i, j=1,2, \ldots, n\right\} .
$$

Then $\mathcal{N}[\mathbf{P}, \mathbf{Q}]$ is called interval matrix, in which $\mathbf{P}$ and $\mathbf{Q}$ are the lower- and upper-bound matrices, respectively.

Based on the lemma given below, the interval matrix $\mathscr{N}[\mathbf{P}, \mathbf{Q}]$ can be converted equivalently to a matrix with normbounded uncertainty. Therefore, the uncertain representation is obtained by seeking a lower-bound matrix $\mathbf{P}$ and an upper-bound matrix $\mathbf{Q}$ such that $\mathscr{A}_{l} \in \mathcal{N}[\mathbf{P}, \mathbf{Q}](l=1$, $2, \ldots, 11)$.
Lemma 1 [34]. The interval matrix $\mathcal{N}[\mathbf{P}, \mathbf{Q}]$ is equivalent to the set $\mathscr{M}_{a}$ defined below.

$$
\mathscr{M}_{a} \triangleq\left\{\mathscr{A} \in \mathbb{R}^{n \times n} \mid \mathscr{A}=\mathbf{A}+\mathbf{G}_{a} \mathbf{F}_{a} \mathbf{E}_{a}, \mathbf{F}_{a}^{\mathrm{T}} \mathbf{F}_{a} \leq \mathbf{I}\right\}
$$

in which matrices $\mathbf{G}_{a} \in \mathbb{R}^{n \times n^{2}}, \mathbf{F}_{a} \in \mathbb{R}^{n^{2} \times n^{2}}$, and $\mathbf{E}_{a} \in \mathbb{R}^{n^{2} \times n}$ are taken as

$$
\begin{aligned}
\mathbf{A} & =\frac{(\mathbf{P}+\mathbf{Q})}{2}, \\
\mathbf{H} & \triangleq\left(h_{i j}\right)_{n \times n}=\frac{(\mathbf{Q}-\mathbf{P})}{2}, \\
\mathbf{G}_{a} & =\left[\sqrt{h_{11} \mathbf{e}_{1}} \cdots \sqrt{h_{1 n} \mathbf{e}_{1}} \cdots \sqrt{h_{n 1} \mathbf{e}_{n}} \cdots \sqrt{h_{n n} \mathbf{e}_{n}}\right], \\
\mathbf{E}_{a} & =\left[\sqrt{h_{11} \mathbf{e}_{1}} \cdots \sqrt{h_{1 n} \mathbf{e}_{n}} \cdots \sqrt{h_{n 1} \mathbf{e}_{1}} \cdots \sqrt{h_{n n} \mathbf{e}_{n}}\right]^{\mathrm{T}}, \\
\mathbf{e}_{i} & =[\underbrace{0, \ldots, 0,1,}_{i} 0, \ldots, 0]^{\mathrm{T}} \in \mathbb{R}^{n}, \quad(i=1,2, \ldots, n), \\
\mathbf{F}_{a} & =\operatorname{diag}\left(\varepsilon_{11}, \ldots, \varepsilon_{1 n}, \ldots, \varepsilon_{n 1}, \ldots, \varepsilon_{n n}\right), \quad\left|\varepsilon_{i j}\right| \leq 1 .
\end{aligned}
$$


Remark 1. The equivalence of $\mathscr{N}[\mathbf{P}, \mathbf{Q}]$ and $\mathscr{M}_{\mathrm{a}}$ has been proved in [34] and thus is omitted here. Readers could verify equalities $\mathbf{P}=\mathbf{A}-\mathbf{G}_{\mathrm{a}} \mathbf{I}_{\mathrm{n}^{2}} \mathbf{E}_{\mathrm{a}}$ and $\mathbf{Q}=\mathbf{A}+\mathbf{G}_{\mathrm{a}} \mathbf{I}_{\mathrm{n}^{2}} \mathbf{E}_{\mathrm{a}}$ for ease of understanding.

With the given matrices $\mathscr{A}_{l}(l=1,2, \ldots, 11)$, by selecting "tight" bound matrices $\mathbf{P}$ and $\mathbf{Q}$ such that $\mathscr{A}_{l} \in \mathcal{N}[\mathbf{P}, \mathbf{Q}]$, matrices $\mathbf{A}, \mathbf{G}_{a}$, and $\mathbf{E}_{a}$ are calculated according to Lemma 1 as

$$
\begin{aligned}
\mathbf{A} & =\left[\begin{array}{cc}
-5.248 & 4.080 \\
1.012 & -4.933
\end{array}\right], \\
\mathbf{G}_{a} & =\left[\begin{array}{cccc}
0.738 & 0.859 & 0 & 0 \\
0 & 0 & 0.632 & 0.737
\end{array}\right], \\
\mathbf{E}_{a} & =\left[\begin{array}{cccc}
0.738 & 0 & 0.632 & 0 \\
0 & 0.859 & 0 & 0.737
\end{array}\right]^{\mathrm{T}} .
\end{aligned}
$$

Similarly, matrices $\mathbf{B}, \mathbf{C}, \mathbf{D}, \mathbf{G}_{i}$, and $\mathbf{E}_{i}(i \in\{b, c, d\})$ in (7) are calculated as

$$
\begin{aligned}
& \mathbf{B}=\left[\begin{array}{ll}
0.631 & 0.389 \\
0.743 & 0.576
\end{array}\right], \\
& \mathbf{G}_{b}=\left[\begin{array}{cccc}
0.061 & 0.160 & 0 & 0 \\
0 & 0 & 0.091 & 0.060
\end{array}\right] \text {, } \\
& \mathbf{E}_{b}=\left[\begin{array}{cccc}
0.061 & 0 & 0.091 & 0 \\
0 & 0.160 & 0 & 0.060
\end{array}\right]^{\mathrm{T}} \text {, } \\
& \mathbf{C}=\left[\begin{array}{cc}
1 & 0 \\
-0.759 & 2.107
\end{array}\right] \text {, } \\
& \mathbf{G}_{c}=\left[\begin{array}{cccc}
0 & 0 & 0 & 0 \\
0 & 0 & 0.425 & 0.455
\end{array}\right], \\
& \mathbf{E}_{c}=\left[\begin{array}{cccc}
0 & 0 & 0.425 & 0 \\
0 & 0 & 0 & 0.455
\end{array}\right]^{\mathrm{T}}, \\
& \mathbf{D}=\left[\begin{array}{cc}
0 & 0 \\
0.034 & 0.700
\end{array}\right] \text {, } \\
& \mathbf{G}_{d}=\left[\begin{array}{cccc}
0 & 0 & 0 & 0 \\
0 & 0 & 0.042 & 0.080
\end{array}\right], \\
& \mathbf{E}_{d}=\left[\begin{array}{cccc}
0 & 0 & 0.042 & 0 \\
0 & 0 & 0 & 0.080
\end{array}\right]^{\mathrm{T}} .
\end{aligned}
$$

Therefore, the set $\mathscr{M}$ encompassing the set $\mathscr{L}$ is obtained to redescribe a group of linear models (6) as a nominal matrix (9) with norm-bounded uncertainties.

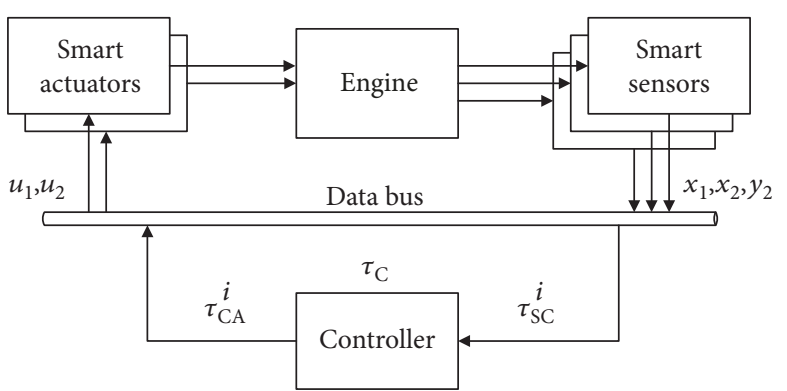

FIgURE 3: Schematic of the NEC system architecture.

\section{Networked Controller Design for the Aeroengine}

In this section, the mathematical expression of the NEC system is presented which takes the transmission delay and the packet dropout into account simultaneously. Then a robust networked engine controller is designed with requirements of robustness against delay/dropout and the satisfactory dynamic performance being met with the NCS stability theory and the regional pole placement, respectively. The former will be presented in Section 3.2 while the latter in Section 3.3. Then by combining linear matrix inequalities (LMIs) from these two theories, the controller design is synthesized in Section 3.4.

3.1. Networked Engine Control System. A schematic of the NEC system architecture is shown in Figure 3, where $\tau_{\mathrm{SC}}^{i}(i=1,2,3), \tau_{\mathrm{CA}}^{i}(i=1,2)$, and $\tau_{\mathrm{C}}$ denote the transmission delay between the sensors and the controller, the transmission delay between the controller and the actuators, and the calculation time of the controller, respectively.

To eliminate the potential tracking error due to reasons like modeling biases or plant disturbances, integrators are incorporated in the controller. Based on [35], by putting integrators at the output $y$, format of the controller is taken as

$$
\delta u(t)=\mathbf{K}_{x} \cdot \delta x(t)+\mathbf{K}_{z} \cdot z(t),
$$

where $z$ is the state of the integrator satisfying

$$
z(t)=\int e(t) d t=\int\left[(\delta y)_{\mathrm{cmd}}-\delta y(t)\right] d t .
$$

Supposed that the sensors are clock driven, the controller and actuators are event driven and the data are transmitted with a single packet and the integrated network-induced delay $\tau$ can be approximated as $\tau=$ $\max _{i}\left(\tau_{\mathrm{SC}}^{i}\right)+\tau_{\mathrm{C}}+\max _{j}\left(\tau_{\mathrm{CA}}^{j}\right)$. When packet dropout occurs, the latest packet is utilized for computation and actuation. 
Then combining (2) with (15) and following the modeling process in [13], the closed-loop system is expressed as

$$
\left\{\begin{array}{c}
\dot{\mathbf{x}}(t)=\mathscr{A} \cdot \delta x(t)+\mathscr{B} \cdot \delta u(t), \\
\delta \mathbf{y}(t)=\mathscr{C} \cdot \delta x(t)+\mathscr{D} \cdot \delta u(t), \\
t \in\left[i_{k} h+\tau_{k}, i_{k+1} h+\tau_{k+1}\right), \\
\delta \mathbf{u}(t)=\mathbf{K}_{x} \cdot \delta x\left(t-\tau_{k}\right)+\mathbf{K}_{z} \cdot z\left(t-\tau_{k}\right), \\
t=i_{k} h+\tau_{k}, i_{k} \in\{1,2,3 \ldots\}, k=1,2, \ldots,
\end{array}\right.
$$

in which $h$ is the sampling period. $\tau_{k}$ is the transmission delay from the sampling instant $i_{k} h$ to the actuating instant $i_{k} h+\tau_{k} \cdot\left\{i_{k}\right\}$ is a nondecreasing sequence satisfying $i_{k+1} \geq i_{k}$ if the data packet disorder is ignored.

More specifically, $i_{k+1}=i_{k}+1$ means that no packet dropout occurs while $i_{k+1}=i_{k}$ means a packet loss in the transmission. By the definition of the symbol $\left\{i_{k}\right\}$, it can be seen that (17) models the transmission delay and the packet dropout simultaneously.

Furthermore, by defining an augmented state vector as $\bar{x} \triangleq\left[\begin{array}{ll}x & z\end{array}\right]^{\mathrm{T}}$ and considering the output reference $(\delta y)$ to be zero here, the closed-loop system (17) can be expressed compactly as

$$
\left\{\begin{aligned}
\dot{\bar{x}}(t) & =\overline{\mathscr{A}} \cdot \delta \bar{x}(t)+\overline{\mathscr{B}} \cdot \delta u(t), \\
t & \in\left[i_{k} h+\tau_{k}, i_{k+1} h+\tau_{k+1}\right), \\
\delta u(t) & =\mathbf{K} \cdot \delta \bar{x}\left(t-\tau_{k}\right), \\
t & =i_{k} h+\tau_{k}, i_{k} \in\{1,2,3, \ldots,\}, k=1,2, \ldots,
\end{aligned}\right.
$$

in which $\mathbf{K} \triangleq\left[\begin{array}{ll}\mathbf{K}_{x} & \mathbf{K}_{z}\end{array}\right] \cdot \overline{\mathscr{A}}$ and $\overline{\mathscr{B}}$ are system matrices that can still be decomposed into a norm-bounded form as

$$
\begin{aligned}
\bar{A} & =\overline{\mathbf{A}}+\Delta \overline{\mathbf{A}}, \\
\bar{B} & =\overline{\mathbf{B}}+\Delta \overline{\mathbf{B}}, \\
{[\Delta \overline{\mathbf{A}}: \Delta \overline{\mathbf{B}}] } & =\overline{\mathbf{G}} \overline{\mathbf{F}}\left[\overline{\mathbf{E}}_{a} ; \overline{\mathbf{E}}_{b}\right]
\end{aligned}
$$

with

$$
\begin{aligned}
\overline{\mathbf{A}} & =\left[\begin{array}{cc}
\mathbf{A} & 0 \\
-\mathbf{C} & 0
\end{array}\right], \\
\overline{\mathbf{B}} & =\left[\begin{array}{c}
\mathbf{B} \\
-\mathbf{D}
\end{array}\right], \\
\overline{\mathbf{G}} & =\left[\begin{array}{cccc}
\mathbf{G}_{a} & 0 & \mathbf{G}_{b} & 0 \\
0 & \mathbf{G}_{c} & 0 & \mathbf{G}_{d}
\end{array}\right], \\
\overline{\mathbf{E}}_{a} & =\left[\begin{array}{cccc}
\mathbf{E}_{a} & \mathbf{E}_{c} & 0 & 0 \\
0 & 0 & 0 & 0
\end{array}\right]^{\mathrm{T}}, \\
\overline{\mathbf{F}} & =\operatorname{diag}\left(\mathrm{F}_{a},-\mathrm{F}_{c}, \mathrm{~F}_{b},-\mathrm{F}_{d}\right), \\
\overline{\mathbf{E}}_{b} & =\left[\begin{array}{llll}
0 & 0 & \mathbf{E}_{b} & \mathbf{E}_{d}
\end{array}\right]^{\mathrm{T}} .
\end{aligned}
$$

The matrices $\overline{\mathscr{A}}, \overline{\mathscr{B}}, \overline{\mathbf{G}}, \overline{\mathbf{E}}_{a}$, and $\overline{\mathbf{E}}_{b}$ can be calculated based on (13) and (14), but they are not listed here due to their high dimensions.

3.2. Stability Theory under Delay/Dropout Condition. In the following, the state feedback controller $\delta u=\mathbf{K} \cdot \delta \bar{x}$ will be designed. Before we start, a frequently used lemma is introduced below which eliminates the uncertain term in LMIs effectively without introducing conservatism. Thus, by this lemma, existing theorems can be extended for uncertain systems straightforwardly.

Lemma 2 [36]. With the given matrices $\mathbf{M}, \mathbf{N}$ with appropriate dimensions, and a symmetric matrix $\mathrm{W}$, the matrix inequality $\quad \mathbf{W}+\mathbf{N}^{\mathrm{T}} \mathbf{F}^{\mathrm{T}} \mathbf{M}^{\mathrm{T}}+\mathbf{M F N}<0$,

holds for all $\mathbf{F}$ satisfying $\mathbf{F}^{\mathrm{T}} \mathbf{F} \leq \mathbf{I}$ if and only if there exists a scalar $\varepsilon>0$ such that

$$
\mathbf{W}+\varepsilon \mathbf{M M}^{\mathrm{T}}+\varepsilon^{-1} \mathbf{N}^{\mathrm{T}} \mathbf{N}<0 .
$$

By Lemma 2, the theorem introduced below guarantees the robustness against delay/dropout for the closed-loop system (18).

Theorem 1 For the given scalars $\eta \geq\left(i_{k+1}-i_{k}\right) \mathrm{h}+\tau_{k+1}$ and $\rho_{j}(j=2,3)$, the closed-loop system (18) is exponentially asymptotically stable with $\mathbf{K}=\mathbf{Y X}^{-1}$ if there exist a scalar $\varepsilon$ $>0$ and matrices $\mathbf{P}>0, \mathbf{T}>0, \mathbf{X}>0, \mathbf{Y}$, and $\mathrm{N}_{i}(i=1,2,3)$ with appropriate dimensions such that (23) holds.

$$
\begin{aligned}
& \Theta\left(\eta, \rho_{2}, \rho_{3}, \mathbf{P}, \mathbf{T}, \mathbf{X}, \mathbf{Y}, \varepsilon\right) \\
& \triangleq\left[\begin{array}{ccccc}
\Omega_{11} & \Omega_{12} & \Omega_{13} & \eta \mathbf{N}_{1} & \left(\overline{\mathbf{E}}_{a} \mathbf{X}\right)^{\mathrm{T}} \\
* & \Omega_{22} & \Omega_{23} & \eta \mathbf{N}_{2} & \left(\overline{\mathbf{E}}_{b} \mathbf{Y}\right)^{\mathrm{T}} \\
* & * & \Omega_{33} & \eta \mathbf{N}_{3} & 0 \\
* & * & * & -\eta \mathbf{T} & 0 \\
* & * & * & * & -\varepsilon \mathbf{I}
\end{array}\right]<0,
\end{aligned}
$$

where

$$
\begin{aligned}
& \Omega_{11}=\operatorname{sym}\left(\mathbf{N}_{1}\right)-\operatorname{sym}(\overline{\mathbf{A}} \mathbf{X})+\varepsilon \overline{\mathbf{G}} \overline{\mathbf{G}}^{\mathrm{T}}, \\
& \Omega_{12}=\mathbf{N}_{2}^{\mathrm{T}}-\mathbf{N}_{1}-\overline{\mathbf{B}} \mathbf{Y}-\rho_{2} \mathbf{X} \overline{\mathbf{A}}^{\mathrm{T}}+\varepsilon \overline{\mathbf{G}} \overline{\mathbf{G}}^{\mathrm{T}} \rho_{2}, \\
& \Omega_{13}=\mathbf{P}+\mathbf{N}_{3}^{\mathrm{T}}+\mathbf{X}-\rho_{3} \mathbf{X} \overline{\mathbf{A}}^{\mathrm{T}}+\varepsilon \overline{\mathbf{G}} \overline{\mathbf{G}}^{\mathrm{T}} \rho_{3}, \\
& \Omega_{22}=-\operatorname{sym}\left(\mathbf{N}_{2}\right)-\operatorname{sym}\left(\rho_{2} \overline{\mathbf{B}} \mathbf{Y}\right)+\varepsilon \rho_{2} \overline{\mathbf{G}} \overline{\mathbf{G}}^{\mathrm{T}} \rho_{2}, \\
& \Omega_{23}=-\mathbf{N}_{3}^{\mathrm{T}}+\rho_{2} \mathbf{X}-\rho_{3} \mathbf{Y}^{\mathrm{T}} \overline{\mathbf{B}}^{\mathrm{T}}+\varepsilon \rho_{2} \overline{\mathbf{G}} \overline{\mathbf{G}}^{\mathrm{T}} \rho_{3}, \\
& \Omega_{33}=\eta \mathrm{T}+2 \rho_{3} \mathbf{X}+\varepsilon \rho_{3} \overline{\mathbf{G}} \bar{G}^{\mathrm{T}} \rho_{3} .
\end{aligned}
$$

Proof. It can be known from [14] that for the given scalars $\eta$ and $\rho_{j}(j=2,3)$, the closed-loop system (18) is exponentially asymptotically stable with $\mathbf{K}=\mathbf{Y X}^{-1}$ if there exist matrices 
$\mathbf{P}>0, \mathbf{T}>0, \mathbf{X}>0, \mathbf{Y}$, and $\mathbf{N}_{i}(i=1,2,3)$ with appropriate dimensions such that (25) holds.

$$
\left[\begin{array}{cccc}
\operatorname{sym}\left(\mathbf{N}_{1}\right)-\operatorname{sym}(\overline{\mathscr{A}} \mathbf{X}) & \mathbf{N}_{2}^{\mathrm{T}}-\mathbf{N}_{1}-\overline{\mathscr{B}} \mathbf{Y}-\rho_{2} \mathbf{X} \overline{\mathscr{A}}^{\mathrm{T}} & \mathbf{P}+\mathbf{N}_{3}^{\mathrm{T}}+\mathbf{X}-\rho_{3} \mathbf{X} \overline{\mathscr{A}}^{\mathrm{T}} & \eta \mathbf{N}_{1} \\
* & -\operatorname{sym}\left(\mathbf{N}_{2}\right)-\operatorname{sym}\left(\rho_{2} \overline{\mathscr{B}} \mathbf{Y}\right) & -\mathbf{N}_{3}^{\mathrm{T}}+\rho_{2} \mathbf{X}-\rho_{3} \mathbf{Y}^{\mathrm{T}} \overline{\mathscr{B}}^{\mathrm{T}} & \eta \mathbf{N}_{2} \\
* & * & \eta \mathbf{T}+2 \rho_{3} \mathbf{X} & \eta \mathbf{N}_{3} \\
* & * & * & -\eta \mathbf{T}
\end{array}\right]<0 .
$$

Then by substituting $[\overline{\mathcal{A}} ; \overline{\mathcal{B}}]=[\overline{\mathbf{A}} \overline{\mathbf{B}}]+\overline{\mathbf{G}} \overline{\mathbf{F}}\left[\overline{\mathbf{E}}_{a} ; \overline{\mathbf{E}}_{b}\right]$ into (25) and using Lemma 2 to matrix $\overline{\mathbf{F}}$, it can be found that (25) holds if there exists a scalar $\varepsilon>0$ satisying (26).

$$
\begin{aligned}
& {\left[\begin{array}{cccc}
\tilde{\Omega}_{11} & \tilde{\Omega}_{12} & \tilde{\Omega}_{13} & \eta \mathbf{N}_{1} \\
* & \tilde{\Omega}_{22} & \tilde{\Omega}_{23} & \eta \mathbf{N}_{2} \\
* & * & \tilde{\Omega}_{33} & \eta \mathbf{N}_{3} \\
* & * & * & -\eta \mathbf{T}
\end{array}\right]+\varepsilon\left[\begin{array}{c}
-\overline{\mathrm{G}} \\
-\rho_{2} \overline{\mathrm{G}} \\
-\rho_{3} \overline{\mathrm{G}} \\
0
\end{array}\right]\left[\begin{array}{c}
-\overline{\mathbf{G}} \\
-\rho_{2} \overline{\mathbf{G}} \\
-\rho_{3} \overline{\mathbf{G}} \\
0
\end{array}\right]^{\mathrm{T}}} \\
& +\varepsilon^{-1}\left[\begin{array}{c}
\left(\overline{\mathbf{E}}_{a} \mathbf{X}\right)^{\mathrm{T}} \\
\left(\overline{\mathbf{E}}_{b} \mathbf{Y}\right)^{\mathrm{T}} \\
0 \\
0
\end{array}\right]\left[\begin{array}{c}
\left(\overline{\mathrm{E}}_{a} \mathbf{X}\right)^{\mathrm{T}} \\
\left(\overline{\mathbf{E}}_{b} \mathbf{Y}\right)^{\mathrm{T}} \\
0 \\
0
\end{array}\right]^{\mathrm{T}}<0,
\end{aligned}
$$

where

$$
\begin{aligned}
& \tilde{\Omega}_{11}=\operatorname{sym}\left(\mathbf{N}_{1}\right)-\operatorname{sym}(\overline{\mathbf{A}} \mathbf{X}), \\
& \tilde{\Omega}_{12}=\mathbf{N}_{2}^{\mathrm{T}}-\mathbf{N}_{1}-\overline{\mathbf{B}} \mathbf{Y}-\rho_{2} \mathbf{X} \overline{\mathbf{A}}^{\mathrm{T}}, \\
& \tilde{\Omega}_{13}=\mathbf{P}+\mathbf{N}_{3}^{\mathrm{T}}+\mathbf{X}-\rho_{3} \mathbf{X} \overline{\mathbf{A}}^{\mathrm{T}}, \\
& \tilde{\Omega}_{22}=-\operatorname{sym}\left(\mathbf{N}_{2}\right)-\operatorname{sym}\left(\rho_{2} \overline{\mathbf{B}} \mathbf{Y}\right), \\
& \tilde{\Omega}_{23}=-\mathbf{N}_{3}^{\mathrm{T}}+\rho_{2} \mathbf{X}-\rho_{3} \mathbf{Y}^{\mathrm{T}} \overline{\mathbf{B}}^{\mathrm{T}}, \\
& \tilde{\Omega}_{33}=\eta \mathbf{T}+2 \rho_{3} \mathbf{X} .
\end{aligned}
$$

Then by Schur complements, (26) is equivalent to (23). The theorem is proved.

Remark 2. In Theorem 1, inequality $\eta \geq\left(i_{k+1}-i_{k}\right) h+\tau_{k+1}$ describes the effect of the transmission delay and packet dropout simultaneously; hence, $\eta$ can be viewed as a delay/ dropout margin.

(i) If no packet drops in the transmission $\left(i_{k+1}=i_{k}+1\right)$, inequality $\tau_{k+1} \leq \eta_{\max }-h$ holds. The maximum allowable transmission delay $\tau_{\max }$ is calculated as

$$
\tau_{\max }=\eta_{\max }-h
$$

(ii) Assuming a constant delay $\tau$ in the transmission, inequality $\left(i_{k+1}-i_{k}\right) \leq\left(\eta_{\max }-\tau\right) / h$ holds. Thus, the maximum allowable number of consecutive packet dropout $n_{\max }$ is calculated as

$$
n_{\max }=\left\lfloor\frac{\eta_{\max }-\tau}{h}\right\rfloor-1
$$

3.3. D-Stability Theory. Though Theorem 1 guarantees that the closed-loop system is exponentially stable, the dynamic performance can still be poor since no constraints are imposed on the rate of decay. To obtain a satisfactory dynamic performance, a simple way is to assign the closedloop poles of the augmented system (18), namely, the eigenvalues of $\overline{\mathscr{A}}+\overline{\mathscr{B}} \mathbf{K}$, to some suitable regions $\mathscr{D}$ on the left-half complex plane. To achieve this, the below is introduced first which is usually referred as the $\mathscr{D}$-stability theory.

Lemma 3 [37]. With the given LMI region $\mathscr{D} \triangleq\{s \in \mathbb{C}$ : $\left.\mathbf{L}+\mathrm{s} \mathbf{M}+\bar{s} \mathbf{M}^{\mathrm{T}}<0\right\}$ and matrix $\mathbf{A}$, the eigenvalues of $\mathbf{A}$ lie in $\mathscr{D}$ if and only if there exists a symmetric matrix $\mathbf{X}>0$ such that

$$
M_{\mathscr{D}}(\mathbf{A}, \mathbf{X}) \triangleq \mathbf{L} \otimes \mathbf{X}+\mathbf{M} \otimes(\mathbf{A X})+\mathbf{M}^{\mathrm{T}} \otimes(\mathbf{A X})^{\mathrm{T}}<0 .
$$

Different types of regions, including the half-open plane, vertical strip area, and conic sector, can be covered by the above LMI description. Other complex regions can also be covered as long as they are convex. To guarantee a minimum decay rate and a minimum damping ratio, in this paper, the disk region $\mathscr{C}(-q, r)$ is chosen as the expected eigenvalue region of which the center is $-q$ and the radius is $r$. This disk region is described by taking

$$
\begin{aligned}
\mathbf{L} & =\left[\begin{array}{cc}
-r & q \\
q & -r
\end{array}\right], \\
\mathbf{M} & =\left[\begin{array}{ll}
0 & 1 \\
0 & 0
\end{array}\right] .
\end{aligned}
$$

Then based on Lemma 3, the theorem below guarantees all the eigenvalues of $\overline{\mathscr{A}}+\overline{\mathscr{B}} \mathbf{K}$ in the disk region $\mathscr{C}(-q, r)$ 
Theorem 2. Given a disk region $\mathscr{C}(-q, r)$ defined by (31), all eigenvalues of $\overline{\mathscr{A}}+\overline{\mathscr{B}} \mathbf{K}$ lie in $\mathscr{C}(-q, r)$ if and only if there exist a symmetric matrix $\mathbf{X}>0$, a matrix $\mathbf{Y}$ with appropriate dimension, and a scalar $\varepsilon>0$ such that

$$
\begin{aligned}
& \Xi(q, r, \mathbf{X}, \mathbf{Y}, \varepsilon) \\
& \triangleq\left[\begin{array}{ccc}
-r \mathbf{X}+\varepsilon \overline{\mathbf{G}} \overline{\mathbf{G}}^{\mathrm{T}} & q \mathbf{X}+\overline{\mathbf{A}} \mathbf{X}+\overline{\mathbf{B}} \mathbf{Y} & 0 \\
* & -r \mathbf{X} & \left(\overline{\mathbf{E}}_{a} \mathbf{X}+\overline{\mathbf{E}}_{b} \mathbf{Y}\right)^{\mathrm{T}} \\
* & * & -\varepsilon \mathbf{I}
\end{array}\right]<0,
\end{aligned}
$$

with the control gain matrix being calculated as $\mathbf{K}=\mathbf{Y X}^{-1}$.

Proof. Being similar to the proof of Theorem 1, by substituting $[\overline{\mathcal{A}} \vdots \overline{\mathcal{B}}]=[\overline{\mathbf{A}} ; \overline{\mathbf{B}}]+\overline{\mathbf{G}} \overline{\mathbf{F}}\left[\overline{\mathbf{E}}_{a} ; \overline{\mathbf{E}}_{b}\right]$ into $M_{\mathscr{D}}(\overline{\mathscr{A}}+\overline{\mathscr{B}} \mathbf{K}$, $\mathbf{X})<0$ and using Lemma 2 to eliminate the uncertain matrix $\overline{\mathbf{F}}, M_{\mathscr{D}}(\overline{\mathscr{A}}+\overline{\mathscr{B}} \mathbf{K}, \mathbf{X})<0$ is equivalent to

$$
\begin{aligned}
& M_{\mathscr{D}}(\overline{\mathbf{A}}+\overline{\mathbf{B}} \mathbf{K}, \mathbf{X})+\varepsilon\left[\begin{array}{ll}
\overline{\mathbf{G}}^{\mathrm{T}} & 0
\end{array}\right]^{\mathrm{T}}\left[\begin{array}{ll}
\overline{\mathbf{G}}^{\mathrm{T}} & 0
\end{array}\right]
\end{aligned}
$$

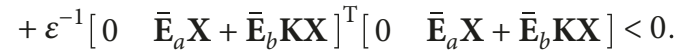

Then by Schur complements and taking $\mathbf{K}=\mathbf{Y X}^{-1}$, is equivalent to (32). The theorem is proved.

3.4. Controller Design Synthesis. Based on the aforementioned work, the controller with strong delay/dropout robustness and satisfactory dynamic performance can be obtained through solving LMIs in Theorem 1 and Theorem 2 simultaneously. These LMIs are written together as

$$
\left\{\begin{array}{l}
\Theta\left(\eta, \rho_{2}, \rho_{3}, \mathbf{P}, \mathbf{T}, \mathbf{X}, \mathbf{Y}, \varepsilon\right)<0, \\
\Xi\left(q, r, \mathbf{X}, \mathbf{Y}, \varepsilon_{1}\right)<0, \\
\mathbf{P}>0 \\
\mathbf{T}>0 \\
\mathbf{X}>0 \\
\varepsilon>0 \\
\varepsilon_{1}>0,
\end{array}\right.
$$

in which $\Theta$ and $\Xi$ are matrices defined in (23) and (32), respectively, and $q, r, \eta$, and $\rho_{j}(j=2,3)$ are predesignated scalars.

Then for the given scalars $q, r$, and $\rho_{j}(j=2,3)$, the maximum delay/dropout margin $\eta_{\max }$ can be calculated by a linear search. Correspondingly, the control gain matrix is calculated as $\mathbf{K}=\mathbf{Y X}^{-1}$.

\section{Application on the Turbofan Aeroengine Simulator}

In this section, the controller design proposed above is applied on the turbofan aeroengine simulator by selecting appropriate closed-loop poles region. The controller is designed based on the uncertain model established in Section 2 and then verified on the simulator itself to validate the applicability of the proposed method.

4.1. Controller Design. Once the uncertain state-space representation is ready, the center $-q$ and the radii $r$ of the disk pole region $\mathscr{C}(-q, r)$ are determined to calculate the control gain matrix. In practical turbofan applications, the percentage overshoot (PO) and the setting time $T_{\text {setting }}$ of the engine control system are usually required to satisfy

$$
\begin{aligned}
\mathrm{PO} & \leq 2 \%, \\
T_{\text {setting }} & \leq 2 \mathrm{~s} .
\end{aligned}
$$

By taking the damping ratio $\xi=0.8$ (dampling angle $\theta=\arccos 0.8)$ and the center $q=6, \mathrm{PO}$ and $T_{\text {setting }}$ are estimated by the formulae of the second-order system as

$$
\begin{aligned}
\mathrm{PO} & =e^{-\pi \xi / \sqrt{1-\xi^{2}} \times 100 \%=1.52 \%,} \\
T_{\text {setting }} & =-\left.\frac{\ln \left(\Delta \times \sqrt{1-\xi^{2}}\right)}{q-r}\right|_{\Delta=0.02}=1.84 \mathrm{~s},
\end{aligned}
$$

in which $r=q \sin \theta$. This fact indicts that the dynamic performance requirements above can be met if $q \geq 6$ and $r \leq$ $0.6 q$. Based on this criterion, by taking $q=8$ and $r=4.8$ and searching $\rho_{j}(j=2,3)$ between $[-10,10]$, the control gain matrix $\mathbf{K}$ is calculated for (18) by solving LMIs in (34) as

$$
\mathbf{K}=\left[\begin{array}{cccc}
-8.829 & -3.244 & 41.298 & -4.678 \\
1.378 & -1.625 & -3.259 & 6.965
\end{array}\right]
$$

The corresponding delay/dropout margin is calculated as $\eta_{\max }=0.104$. Then according to (18), the control gain matrix $\mathbf{K}$ is implemented in the form of

$$
\begin{aligned}
\mathbf{K}_{x} & =\left[\begin{array}{cc}
-8.829 & -3.244 \\
1.378 & -1.625
\end{array}\right], \\
\mathbf{K}_{z} & =\left[\begin{array}{ll}
41.298 & -4.678 \\
-3.259 & 6.965
\end{array}\right] .
\end{aligned}
$$

In the NEC system, the transmission delay $\tau$ is bounded in one sampling period $h$ if a time-triggered data bus is utilized [23]. Therefore, taking $\tau$ as its worst value $h=0.02 \mathrm{~s}$, $\eta_{\max }=0.104$ indicates a maximum allowable number of consecutive packet dropout $n_{\max }=3$ based on Remark 2, namely, the NEC system is stable under a consecutive packet dropout being not severer than 3 in every 4 sampling cycles when the time delay $\tau=0.02 \mathrm{~s}$.

4.2. Simulation. For comparison, another controller $\mathbf{K}_{\text {without } \Delta}$ is calculated under the same condition but without 

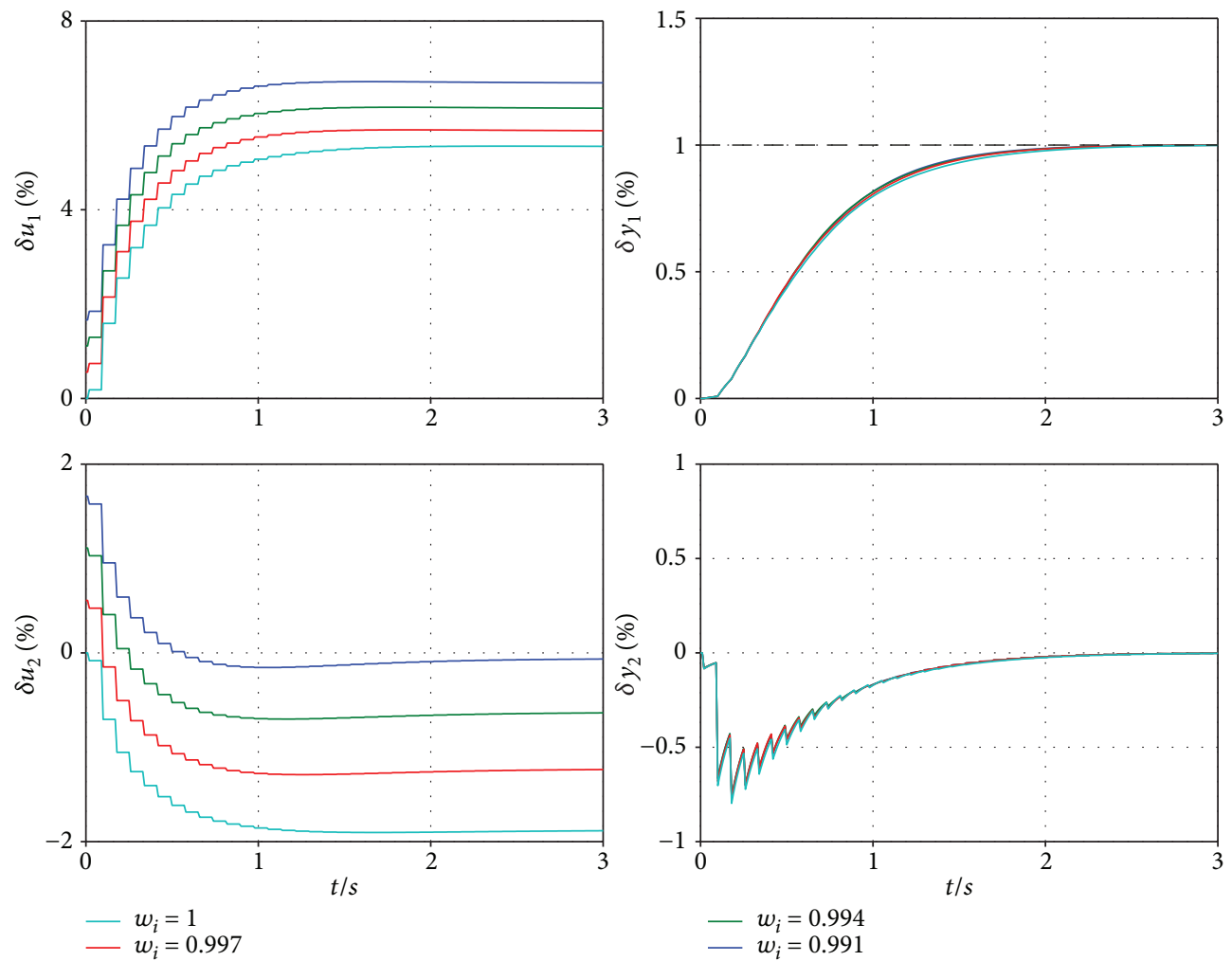

Figure 4: Step response under various deteriorations with $\mathbf{K}$.

considering the uncertainties. Comparison is performed between these two controllers to verify the effectiveness of our design.

By applying them on the component-level aeroengine simulator and varying the degree of deterioration $w$, unit step response simulations are conducted on the output $y_{1}$ as shown in Figures 4 and 5. In each simulation, the health parameter $w_{i}(i=1, \ldots, 8)$ is taken as $\{1,0.997,0.994$, $0.991\}$, while the network condition is taken as the extreme situation $\tau=0.02 \mathrm{~s}$ and $n_{\max }=3$ as discussed above. The control input $u$ and the output $y$ in these figures have already been normalized by their designed values $u_{\text {designed }}$ and $y_{\text {designed }}$.

Comparing Figure 4 (with uncertainties) with Figure 5 (without uncertainties), one can see that the settling time in Figure 4 is smaller than that in Figure 5. This shows that the controller design that is taking uncertainties into consideration is more robust and has a smaller settling time than the one without considering the uncertainties. Besides, it can be observed from Figure 4 that

(1) The NEC system remains stable under the transmission delay and packet dropout described by the delay/ dropout margin $\eta_{\max , \mathscr{C}(-8,4.8)}=0.104$

(2) The output $y_{1}$ presents a satisfactory as well as a consistent dynamic performance under different deteriorations. Besides, the response curves do not overshoot significantly since the disk region has a small imaginary part (damping angle $\theta=\arcsin 0.6$ )
(3) The output could track the reference command without the steady-state error

From the points listed above, the robust networked controller designed in this paper has achieved the expected goal, namely, the three control requirements proposed in Introduction.

4.3. Discussions. In this section, discussions are extended on the relationship between the delay/dropout margin and the disk region $\mathscr{C}(-q, r)$. Besides, the conservatism which is introduced when obtaining the norm-bounded uncertainties is also discussed.

4.3.1. Relationship between the Delay/Dropout Margin and the Disk Region. As mentioned above, the expected disk region $\mathscr{C}(-q, r)$ should satisfy $q \geq 6$ and $r \leq 0.6 q$. Therefore, by varying the center $q \in[6,20]$ and taking the radii $r$ as $r=$ $0.6 q$ for a constant damping ratio, different gain matrices $K_{\mathscr{C}(-q, r)}$ are and their delay/dropout margins $\eta_{\max }$ are calculated in Table 3.

From Table 3, it also can be observed that the delay/ dropout margins decrease rapidly while the pole region is being far away from the imaginary axis. Therefore, there should be a trade-off between the robustness against delay/ dropout and the dynamical performance when designing the controller.

4.3.2. Conservatism Introduced in the Modeling Process. In this paper, a data-based method is applied to obtain the 

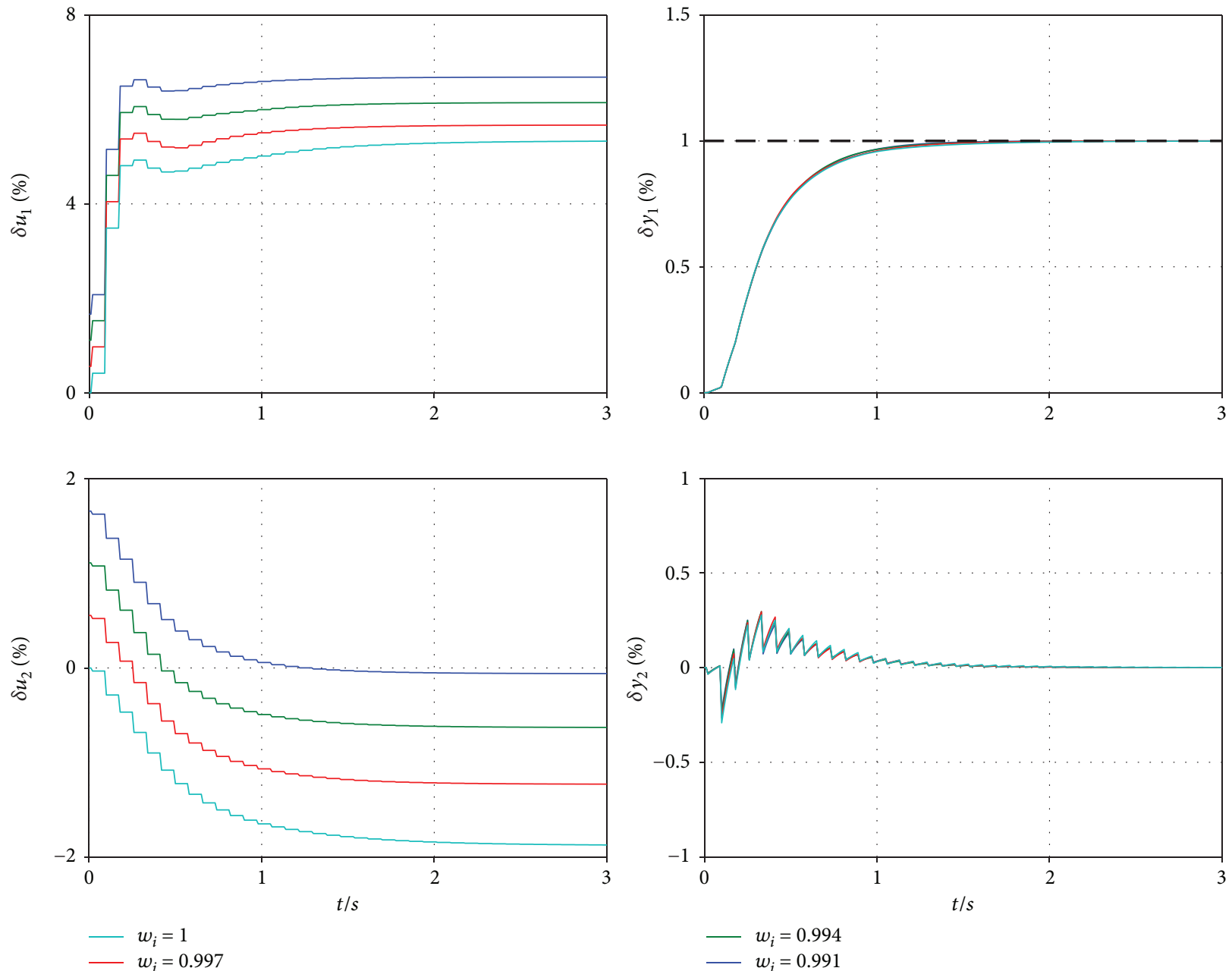

FIGURE 5: Step response under various deteriorations with $\mathbf{K}_{\text {withouts }}$
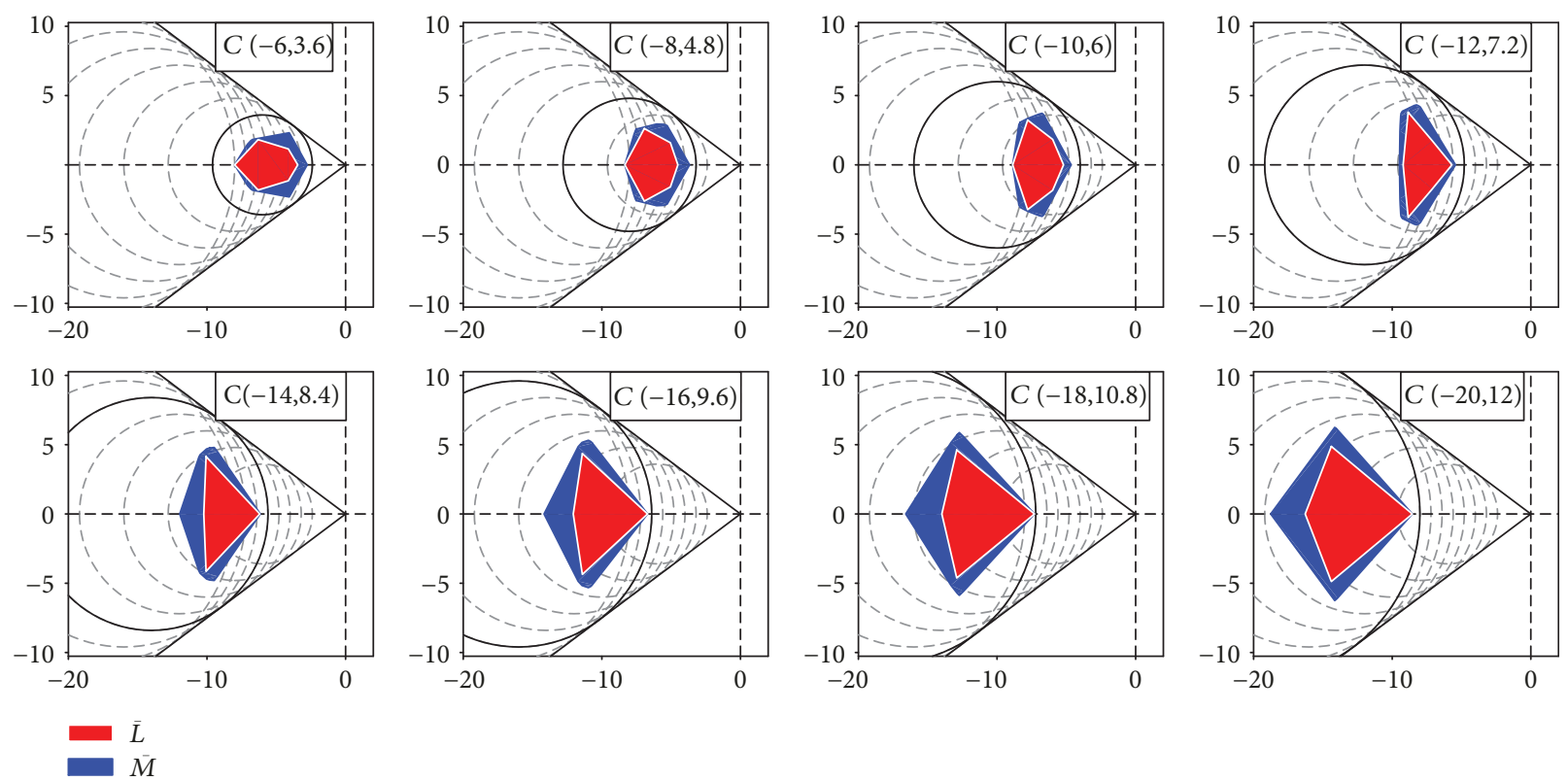

Figure 6: Convex hull of the closed-loop poles under different control gain matrices $K_{\mathscr{C}(-q, r)}$ 
norm-bounded uncertainties in Section 2.3. By this method, a group of system matrices $(\mathscr{L}$ in (6)) is redescribed as a nominal matrix with norm-bounded uncertainties $(\mathscr{M}$ in (9)). However, conservatism is introduced in this modeling process since $\mathscr{L}$ is only a subset of $\mathscr{M}$. To demonstrate the conservatism graphically, integrators are incorporated as mentioned in Section 3.1 to augment $\mathscr{L}$ and $\mathscr{M}$ to $\overline{\mathscr{L}}$ and $\bar{M}$ defined below as

$$
\begin{aligned}
& \bar{L} \triangleq\left\{\left[\bar{A}_{1}: \bar{B}_{1}\right], \cdots,\left[\bar{A}_{10}: \bar{B}_{11}\right]\right\} \text { with } \\
& \bar{A}_{1} \triangleq\left[\begin{array}{cc}
A_{1} & 0 \\
-C_{1} & 0
\end{array}\right], \\
& \bar{B}_{1} \triangleq\left[\begin{array}{c}
B l \\
-D_{l}
\end{array}\right](l=1,2, \ldots, 11)
\end{aligned}
$$

and

$$
\begin{aligned}
\bar{M} \triangleq & \{[\bar{A} \vdots \bar{B}] \mid[\bar{A} ; \bar{B}]=[\overline{\mathbf{A}}: \overline{\mathbf{B}}] \\
& \left.+\overline{\mathbf{G}} \overline{\mathbf{F}}\left[\overline{\mathbf{E}}_{a}: \overline{\mathbf{E}}_{b}\right], \overline{\mathbf{F}}^{\mathrm{T}} \overline{\mathbf{F}} \leq \mathbf{I}\right\}
\end{aligned}
$$

in which $\overline{\mathscr{M}}$ is exactly the augmented system matrices in (18).

Based on the gain matrices of various disk regions obtained in the previous section, the closed-loop poles (namely, the eigenvalues of $\left.\overline{\mathscr{A}}+\overline{\mathscr{B}} \mathbf{K}_{\mathscr{C}(-q, r)}\right)$ are calculated for $\overline{\mathscr{L}}$ and $\overline{\mathscr{M}}$. As a result, the convex hulls of these poles are drawn in Figure 6, in which the pole region of $\overline{\mathscr{L}}$ is denoted in red while the counterpart of $\overline{\mathscr{M}}$ is denoted in blue. It can be seen that certain conservatism is introduced but is still at an acceptable level.

4.3.3. Conservatism Introduced in the Controller Design Process. The conservatism is not just introduced by the modeling but also introduced by the controller. In this subsection, we combine the networked controller design theory [13] with the $\mathscr{D}$-stability theorem to design a controller for the uncertain system (18) as a comparison.

By using the same disk region selected in this paper and taking the lower bound of time delay $\tau_{m}$ as 0 , the maximum allowable value of $\eta_{\max }$ is calculated as 0.112 based on [13]. By contrast, $\eta_{\max }$ calculated in this paper is 0.104 . Considering a dropout-free transmission, the gap means a larger maximum allowable delay $\tau_{\max }$ by $8 \mathrm{~ms}$ which is small enough. Therefore, only a little conservatism is introduced in the controller design process.

However, it should be pointed out that the theorem in [13] is in the form of bilinear matrix inequality (BMI), which has to be solved by a complex iterative algorithm. In contrast, the method proposed in this paper is numerically tractable. This makes the controller much easier to be calculated with existing LMI toolbox.

\section{Conclusion}

In this paper, efforts are made to establish the uncertain description for aeroengines with aging and deterioration. Based on this model, the controller with both strong delay/ dropout robustness and satisfactory dynamic performance is designed through solving linear matrix inequalities (LMIs). The validity and applicability of this approach are elaborated through simulations under the deteriorated and networked conditions. The corresponding delay/dropout margin is also calculated, which provides a reference for future development of the aeroengine-distributed control system. Admittedly, with some degree of conservatism, the controller design approach presented in this paper offered a feasible and practical mean to obtain a robust networked controller

\section{Data Availability}

Data can be provided if needed.

\section{Conflicts of Interest}

The authors declare that they have no conflicts of interest.

\section{Acknowledgments}

The authors would like to thank Assoc. Prof. Jiwei Wen in Jiangnan University and Mister Qian Feng in The University of Auckland for their beneficial suggestions.

\section{References}

[1] D. E. Culley, R. Thomas, and J. Saus, "Concepts for distributed engine control," in 43rd AIAA/ASME/SAE/ASEE Joint Propulsion Conference \& Exhibit, p. 5709, Cincinnati, OH, USA, July 2007.

[2] J. A. DeCastro, C. A. Palmer, and A. Behbahani, "Meeting the requirements of distributed engine control via decentralized, modular smart sensing," in 46th AIAA/ASME/SAE/ ASEE Joint Propulsion Conference \& Exhibit, p. 6748, Nashville, TN, USA, July 2010.

[3] O. A. Watts, L. Frediani, and M. W. Usrey, “Technical opportunities for high temperature smart $\mathrm{p} 3$ sensors and electronics for distributed engine control," in 52nd AIAA/SAE/ASEE Joint Propulsion Conference, p. 4512, Salt Lake City, UT, USA, 2016.

[4] A. Nasiri, S. K. Nguang, and A. Swain, "Adaptive sliding mode control for a class of mimo nonlinear systems with uncertainties," Journal of the Franklin Institute, vol. 351, no. 4, pp. 2048-2061, 2014.

[5] L. Ruichao, G. Yingqing, S. K. Nguang, and C. Yifeng, “Takagisugeno fuzzy model identification for turbofan aero-engines with guaranteed stability," Chinese Journal of Aeronautics, vol. 31, no. 6, pp. 1206-1214, 2018.

[6] T. M. Seitz, O. B. Macmann, A. R. Behbahani, and F. Khoury, "Development of distributed control systems for aircraft turbofan engines," in 52nd AIAA/SAE/ASEE Joint Propulsion Conference, p. 4805, Salt Lake City, UT, USA, 2016.

[7] G. L. Thomas, D. E. Culley, and A. Brand, "The application of hardware in the loop testing for distributed engine control," in 52nd AIAA/SAE/ASEE Joint Propulsion Conference, p. 4654, Salt Lake City, UT, USA, 2016.

[8] Y. Wei, J. Qiu, and H.-K. Lam, “A novel approach to reliable output feedback control of fuzzy-affine systems with time delays and sensor faults," IEEE transactions on fuzzy systems, vol. 25, no. 6, pp. 1808-1823, 2017. 
[9] Y. Wei, J. Qiu, P. Shi, and M. Chadli, "Fixed-order piecewiseaffine output feedback controller for fuzzy-affine-model-based nonlinear systems with time-varying delay," IEEE Transactions on Circuits and Systems I: Regular Papers, vol. 64, no. 4, pp. 945-958, 2017.

[10] J. Zhang, P. Shi, J. Qiu, and S. K. Nguang, "A novel observerbased output feedback controller design for discrete-time fuzzy systems," IEEE Transactions on Fuzzy Systems, vol. 23, no. 1, pp. 223-229, 2015.

[11] A. Behbahani, M. Usrey, and Y. Liu, "High-temperature sensor and electronics packaging technologies for distributed engine controls," in 50th AIAA/ASME/SAE/ASEE Joint Propulsion Conference, p. 3533, Cleveland, OH, USA, 2014.

[12] M. Pakmehr, M. Dhingra, N. Fitzgerald et al., "Distributed architectures integrated with high-temperature electronics for engine monitoring and control," in 47th AIAA/ASME/ SAE/ASEE Joint Propulsion Conference \& Exhibit, p. 6148, San Diego, CA, USA, July-August 2011.

[13] X. Jiang, Q. L. Han, S. Liu, and A. Xue, "A new $H_{\infty}$ stabilization criterion for networked control systems," IEEE Transactions on Automatic Control, vol. 53, no. 4, pp. 1025-1032, 2008.

[14] D. Yue, Q.-L. Han, and C. Peng, "State feedback controller design of networked control systems," in Proceedings of the 2004 IEEE International Conference on Control Applications, 2004, pp. 242-247, Taipei, Taiwan, September 2004.

[15] M. Donkers, W. Heemels, N. Van de Wouw, and L. Hetel, "Stability analysis of networked control systems using a switched linear systems approach," IEEE Transactions on Automatic Control, vol. 56, no. 9, pp. 2101-2115, 2011.

[16] A. Kruszewski, W.-J. Jiang, E. Fridman, J. P. Richard, and A. Toguyeni, "A switched system approach to exponential stabilization through communication network," IEEE transactions on control systems technology, vol. 20, no. 4, pp. 887900, 2012.

[17] W.-A. Zhang and L. Yu, "Modelling and control of networked control systems with both network-induced delay and packet-dropout," Automatica, vol. 44, no. 12, pp. 32063210, 2008.

[18] W.-A. Zhang and L. Yu, "A robust control approach to stabilization of networked control systems with time-varying delays," Automatica, vol. 45, no. 10, pp. 2440-2445, 2009.

[19] D. Huang and S. K. Nguang, "Robust control for uncertain networked control systems with random delays," in Lecture Notes in Control and Information Sciences, vol. 386, Springer, London, 2009.

[20] J. Wen, L. Peng, and S. K. Nguang, "Finite-time control for discrete-time markovian jump systems with deterministic switching and time-delay," International journal of control, automation and systems, vol. 12, no. 3, pp. 473-485, 2014.

[21] R. K. Belapurkar, Stability and Performance of Propulsion Control Systems with Distributed Control Architectures and Failures, [Ph. D. thesis], The Ohio State University, 2012.

[22] R. Li, Y. Guo, Y. Chen, and Z. Zhao, "Networked guaranteed cost control for turbofan aero-engines," in 2017 36th Chinese Control Conference (CCC), pp. 251-256, Dalian, China, July 2017.

[23] R. K. Yedavalli, R. K. Belapurkar, and A. Behbahani, "Design of distributed engine control systems for stability under communication packet dropouts," Journal of Guidance, Control, and Dynamics, vol. 32, no. 5, pp. 1544-1549, 2009.
[24] J. Kratz and R. K. Yedavalli, "A riccati equation based robust control design with application to a gas turbine engine leading toward distributed control," in 50th AIAA/ASME/SAE/ASEE Joint Propulsion Conference, p. 3639, Cleveland, OH, USA, 2014.

[25] T. Seitz, R. Yedavalli, O. Macmann, and A. Behbahani, "Robust control of turbine engines using distributed networks with multiple time delays," Journal of guidance, control, and dynamics, vol. 40, no. 11, pp. 2771-2781, 2017.

[26] S. K. Nguang and W. Assawinchaichote, " $\mathrm{H}_{\infty}$ filtering for fuzzy dynamical systems with D stability constraints," IEEE Transactions on Circuits and Systems I: Fundamental Theory and Applications, vol. 50, no. 11, pp. 1503-1508, 2003.

[27] S. K. Nguang and P. Shi, "Robust $H_{\infty}$ output feedback control design for fuzzy dynamic systems with quadratic $D$ stability constraints: an LMI approach," Information Sciences, vol. 176, no. 15, pp. 2161-2191, 2006.

[28] S.-G. Zhang, Y.-Q. Guo, and J. Lu, "Research on aircraft engine component-level models based on gas Turb/MATLAB," Journal of aerospace power, vol. 27, no. 12, pp. 2850-2856, 2012.

[29] J. Kurzke, "How to create a performance model of a gas turbine from a limited amount of information," in ASME Turbo Expo 2005: Power for Land, Sea, and Air, pp. 145-153, Reno, Nevada, USA, June 2005.

[30] J. Kurzke, "About simplications in gas turbine performance calculations," in ASME Turbo Expo 2007: Power for Land, Sea, and Air, pp. 493-501, Montreal, Canada, May 2007.

[31] L. C. Jaw and J. D. Mattingly, Aircraft Engine Controls: Design, System Analysis, and Health Monitoring, American Institute of Aeronautics and Astronautics, 2009.

[32] J. Lu, Y.-q. GUO, and X.-l. CHEN, "Establishment of aeroengine state variable model based on linear fitting method," Journal of aerospace power, vol. 26, no. 5, pp. 1172-1177, 2011.

[33] R. E. Moore, R. B. Kearfott, and M. J. Cloud, Introduction to Interval Analysis, Society for Industrial and Applied Mathematics, Siam, 2009.

[34] F. Wu, Z. Shi, and G. Dai, "On robust stability of dynamic interval systems," Control Theory \& Applications, vol. 18, no. 1, pp. 113-115, 2001.

[35] G. C. Goodwin, S. F. Graebe, and M. E. Salgado, Control System Design, vol. 240, Prentice Hall, Upper Saddle River, NJ, USA, 2001.

[36] L. Xie, M. Fu, and C. E. de Souza, " $H_{\infty}$ control and quadratic stabilization of systems with parameter uncertainty via output feedback," IEEE Transactions on Automatic Control, vol. 37, no. 8, pp. 1253-1256, 1992.

[37] M. Chilali and P. Gahinet, " $H_{\infty}$ design with pole placement constraints: an LMI approach," IEEE Transactions on Automatic Control, vol. 41, no. 3, pp. 358-367, 1996. 


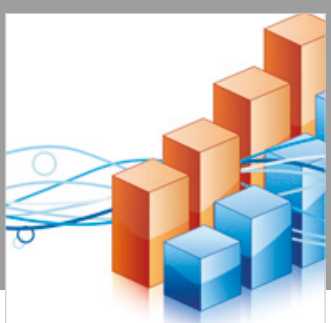

Advances in

Operations Research

\section{-n-m}
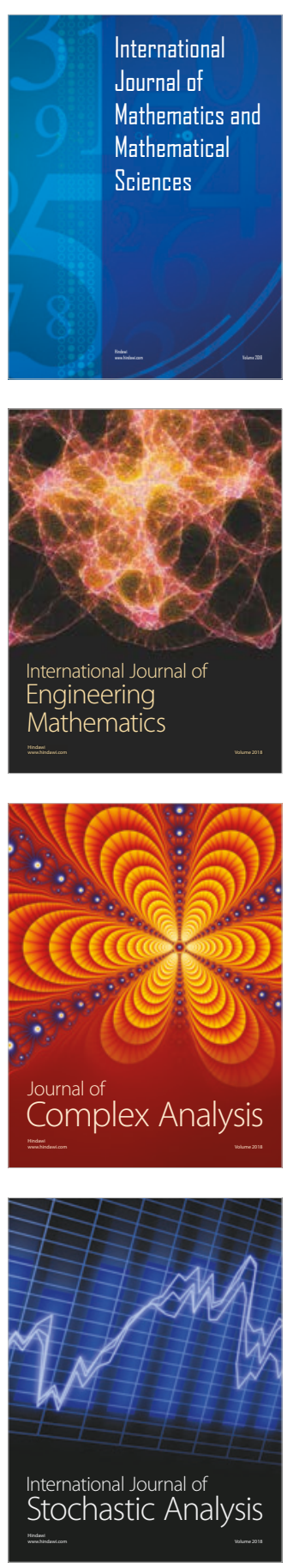
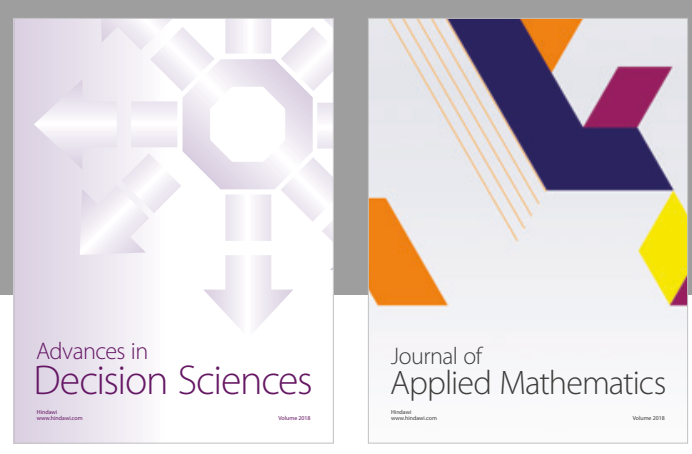

Journal of

Applied Mathematics
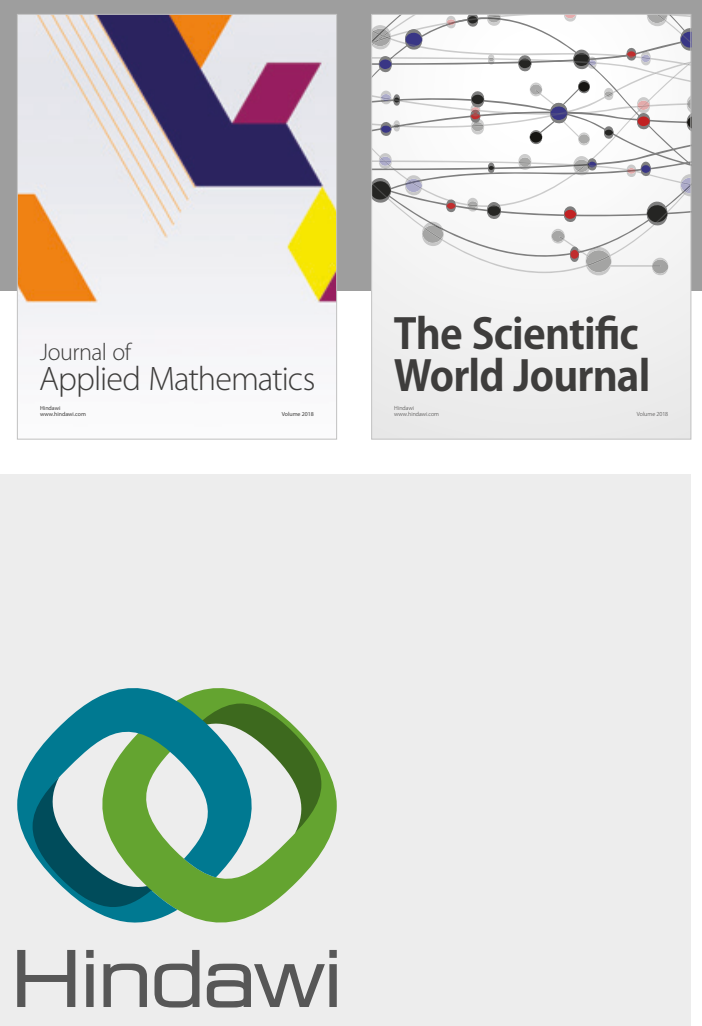

Submit your manuscripts at

www.hindawi.com

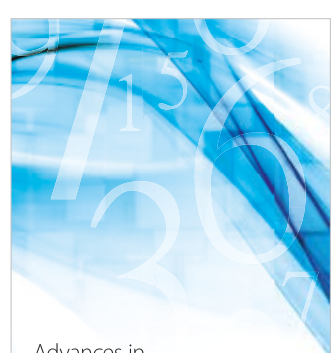

Advances in
Numerical Analysis
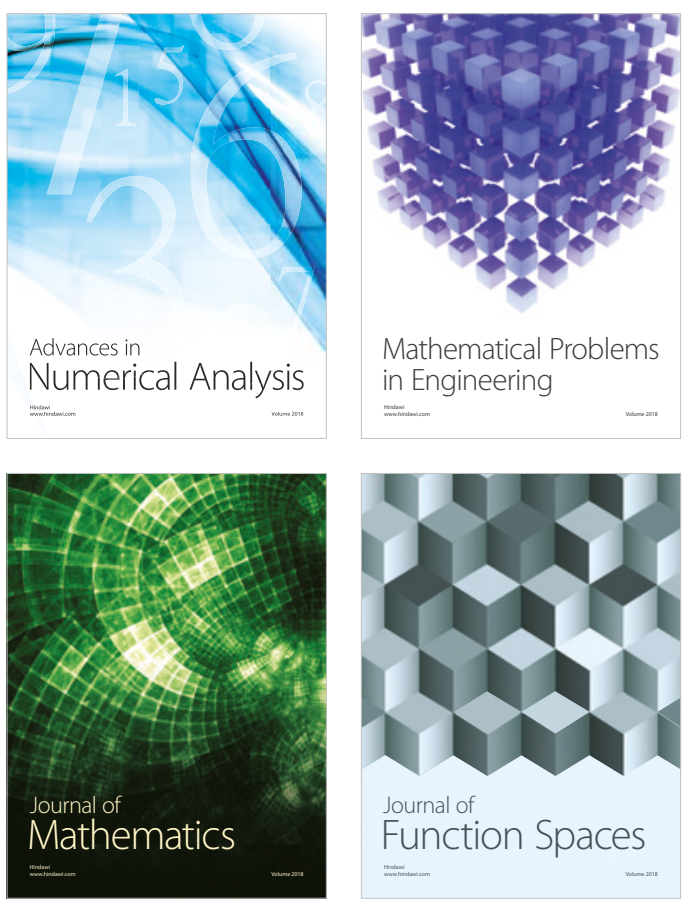

Mathematical Problems in Engineering

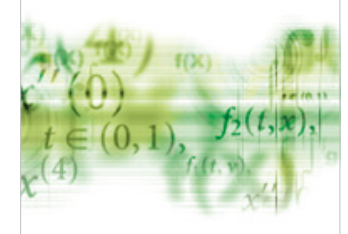

International Journal of

Differential Equations

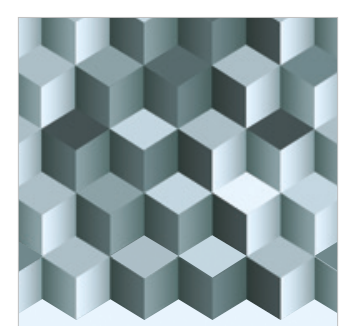

Journal of

Function Spaces
The Scientific

World Journal

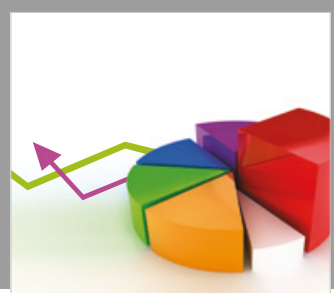

Journal of

Probability and Statistics
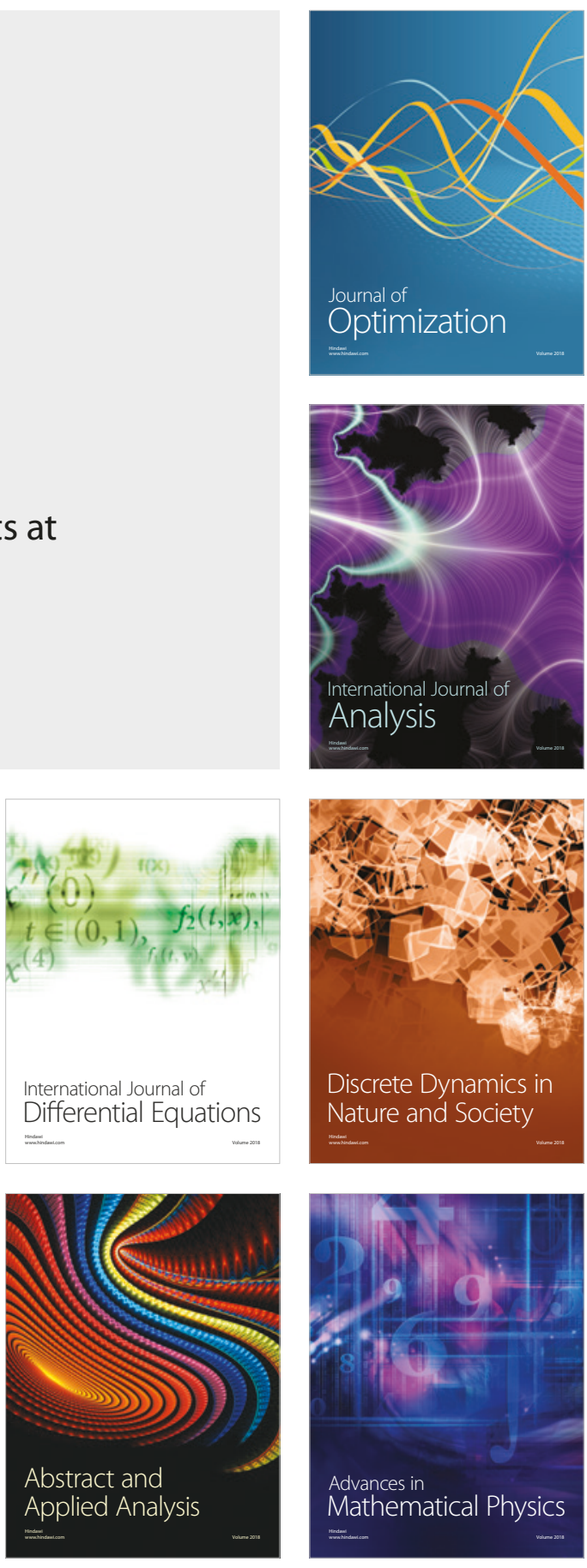